\title{
The Effect of Umbilical Cord Blood Mesenchymal Stem Cells on the Neurological Function and the Expression of Caspase-3 in Cerebral Ischemia- reperfusion Rats
}

\author{
Ming Zhang ${ }^{1 \#}$, Xiaomin Hua ${ }^{2 \#}$, Yudan Zhao ${ }^{2}$, Bin Wang ${ }^{2 *}$ and Yujun Xia ${ }^{1 *}$ \\ ${ }^{1}$ Department of Anatomy, Qingdao University Medical College, Qingdao 266071, People's Republic of China \\ ${ }^{2}$ Department of Microbiology, Qingdao University Medical College, Qingdao 266071, People's Republic of China \\ \#These authors Contributed Equally to the work
}

\begin{abstract}
Cerebral infarction, a type of ischemic stroke disease, is a threat to human health. Thrombolytic therapy and brain protection methods have been used to treat cerebral infarction clinically. Recently, cell therapy has become a hotspot. This study aimed to explore the effect of Umbilical Cord Blood Mesenchymal Stem Cells on the neurological function and the expression of caspase- 3 in cerebral ischemia-reperfusion rats.

Methods: The rat cerebral infarction models were established. The treatment group was injected into $2 \times 106 \mathrm{Um}$ bilical Cord Blood Mesenchymal Stem Cells; Sham-operated group and the control were injected into the same amount of cell culture media. Evaluation of neurological function was performed and the mRNA and protein expression level of caspase-3 was detected using RT-PCR and immunohistochemical method, respectively. Apoptosis analysis was assessed using a TUNEL kit.

Results: Labeled Umbilical Cord Blood Mesenchymal Stem Cells survived well after being injected into rats, while the green fluorescence signal was not discovered in the control and sham-operated group; The neurological function of rats in the treatment group improved compared to the control $(p<0.05)$. RT-PCR results indicated that the expression level of caspase-3 significantly decreased in rats of the treatment group $(P<0.01)$, while there was no significant difference between the sham-operated group and the control $(P>0.05)$. Immunohistochemistry results indicated that the number of caspase- 3 positive cells decreased in the treatment group than the control $(P<0.01)$, while there was no significant difference between the sham-operated group and the control $(P>0.05)$. Apoptosis results showed that TUNEL-positive cells were significantly lower in the treatment group than the control group. This study indicated that Umbilical Cord Blood Mesenchymal Stem Cells contribute to the recovery of neurological function of cerebral ischemia-reperfusion rats and the expression of caspase- 3 decreased.
\end{abstract}

Keywords: Ligand; Ion; Cu; Determination; Reagent

\section{Introduction}

Cerebral infarction, accounting for $75 \%$ of cerebrovascular disease, is a threat to human health, and the mortality and disability rates are very high [1]. Recently, cell therapy has been a hotspot. However, many ethical issues, including the obtaining and culturing of neural stem cells, limit its application clinically. Umbilical Cord Blood Mesenchymal Stem Cells (UCB-MSCs) belong to pluripotent stem cell and improved the limb function in cerebral ischemia-reperfusion rats $[2,3]$. Studies have shown that UCB-MSCs are capable of amplification in vitro compared to the bone marrow-derived mesenchymal stem cells (BM-MSCs) [4,5]. UCB-MSCs have been the seed cells to be injected to treat cerebral infarction. The purpose of this study is to explore the effect of UCB-MSCs on the neurological function and the expression of caspase- 3 in cerebral infarction rats.

\section{Materials and Methods}

\section{Rats}

All procedures described in the study were reviewed and approved by the Ethical Committee of Qingdao University, Qingdao City, China. Wistar, a widely used experimental strain, male, 2-month-old, clean grade, weight $(250 \pm 20) \mathrm{g}$, were provided by Qingdao Food and Drug Administration.

\section{Grouping}

81 wistar rats were divided into three groups randomly: UCB-
MSCs Group (the treatment group), sham-operated group and the control group. Each group was divided into three subgroups: 1d, 3d, 7d, containing 9 rats per subgroup. 24 hours after the successful modeling, UCB-MSCs $\left(1 \times 10^{7} / \mathrm{mL}\right)$ provided by Tsinghua university, were injected into rats. Sham-operated group was not treated.

\section{Establishment of rat cerebral ischemia-reperfusion model}

Rat cerebral ischemia-reperfusion model was established according to Longa's methods [6]. After the animal regains total consciousness, neurological deficits can be evaluated by a simple scale (five-point scale) as follows:

0 : no observable deficits

*Corresponding authors: Yujun Xia, Department of Anatomy, Qingdao University Medical College, Qingdao 266071, People's Republic of China, Tel: +86-13863976458; E-mail: Xiayujun62@163.com

Bin Wang, Department of Microbiology, Qingdao University Medical College, Qingdao 266071, People's Republic of China, Tel: +86-13685326203; E-mail: wangbin532@126.com

Received: March 23, 2015; Accepted: April 16, 2015; Published April 20, 2015

Citation: Zhang M, Hua X, Zhao Y, Xia Y, Wang B (2015) The Effect of Umbilical Cord Blood Mesenchymal Stem Cells on the Neurological Function and the Expression of Caspase-3 in Cerebral Ischemia-reperfusion Rats. Biochem Anal Biochem 4: 170. doi:10.4172/2161-1009.1000170

Copyright: (c) 2015 Zhang M, et al. This is an open-access article distributed unde the terms of the Creative Commons Attribution License, which permits unrestricted use, distribution, and reproduction in any medium, provided the original author and source are credited. 
1: failure to extend contralateral forepaw (mild focal neurologic deficit)

2: circling in a direction contralateral to infarct (moderate focal neurologic deficit)

3: falling in a direction contralateral to infarct (severe focal neurologic deficit)

4: depressed level of consciousness without spontaneous movement

Scoring 2 and 3 were collected for subsequent trials. The right carotid artery of sham-operated group was exposed and sutured later.

\section{Immunohistochemistry}

After rats were anesthetized, brain tissues were sliced and the sections were chosen for immunohistochemical staining. Samples were then incubated with the primary antibodies followed by incubation with a secondary antibody (Bioss, China) for 30 minutes. The reaction was visualized using diaminobenzidine (DAB). Nikon ECLIPSE E600 computer was used for image analysis. The percentage of caspase-3positive cells from each sample was calculated.

\section{RT-PCR analysis}

Total RNA was isolated and reverse-transcribed using RNA isolation Kit (Cwbiotech, China) and Real Time PCR Kits (Takara, China) according to the manufacturer's instructions, respectively. PCR was performed using the following PCR primers: F-TGGAACAAATGGACCTGTTGACC-3, R-AGGACTCAAATTCTGTTGCCACC-3. Semi-quantitative analysis was performed using ID Image Analysis software.

\section{Apoptosis analysis}

Apoptotic cells in frozen brain tissues were detected using DeadEnd ${ }^{\mathrm{TM}}$ Fluorometric TUNEL System (Promega, USA) according to the manufacturer's instructions.

\section{Statistical analysis}

All statistical analysis was performed using SPSS 11.5. All data were performed using Student's t-test and one-way ANOVA. $\mathrm{P}<0.05$ was considered statistically significant.

\section{Results}

\section{Labeled UCB-MSCs distributed in hippocampal CA1 region}

Green fluorescence labeled UCB-MSCs were visible in hippocampal CA1 region under the microscope, suggesting that UCB-MSCs were injected into the rat brain tissues of the treatment group and survived well, while the green fluorescence signal was not discovered in the control and sham-operated group (Figure 1).

\section{Comparison of neurological function}

Neurological dysfunction was not found in the sham-operated group, while there was apparent limb dysfunction in the control group, which revived mostly 2 hours after the operation. Compared to the control group, there was no significant improvement in the $1 \mathrm{~d}$ treatment group $(\mathrm{P}>0.05)$. The score decreased in the $7 \mathrm{~d}$ surgery group $(1.333 \pm$ $0.816)$ than the control group $(2.167 \pm 0.408)$, and the difference was statistically significant $(\mathrm{P}<0.05)$ (Figure $2 \mathrm{~A})$.

The effect of UCB-MSCs on the mRNA expression level of caspase- 3 in ischemia-reperfusion rats

The mRNA expression level of caspase-3 in subgroups of the

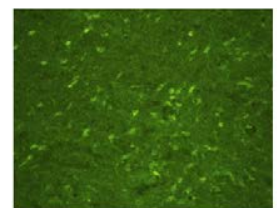

a

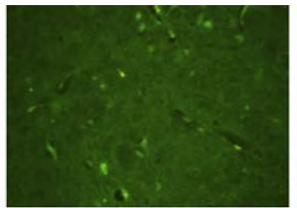

b

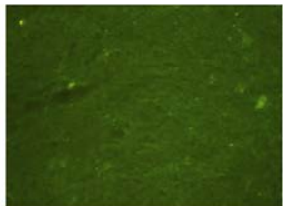

c
Figure 1: Green fluorescence labeled UCBMSCs distributed in rat hippocampal CA1 region of the treatment group $(\times 100)(a: 1 d ; b: 3 d$; c: $7 d)$.

\section{A}

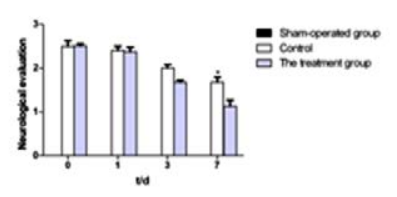

C

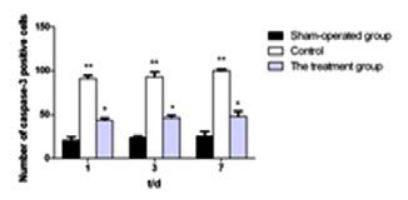

B

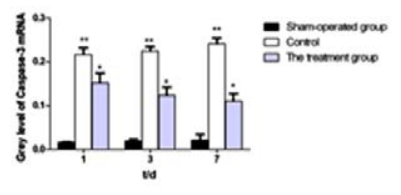

D

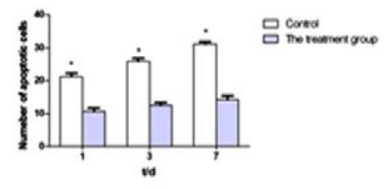

Figure 2: (a) Comparison of neurological function in three groups; (b) Grey level of caspase-3 mRNA in three groups; (c) Comparison of caspase-3 immunohistochemical staining positive cells; $d$ Comparison of TUNEL-positive cells in the treatment group and the control.
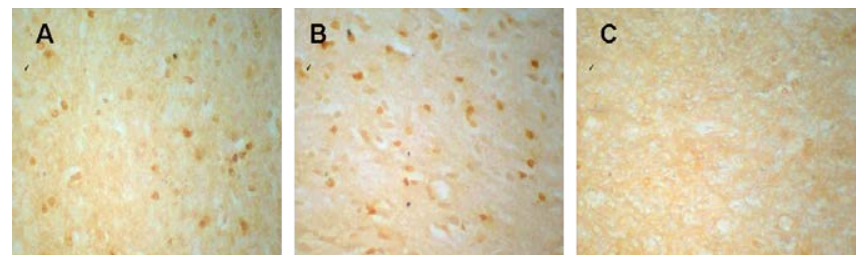

Figure 3: Immunohistochemical staining of caspase-3 and the caspase-3 positive cells were observed $(\times 100)(a$ : the control group; b: the treatment group; c: the sham-operated group)

treatment group decreased compared to the control group, and the difference was statistically significant $(\mathrm{P}<0.01)$ (Figure $2 \mathrm{~B}$ ).

\section{Immunohistochemistry analysis}

Immunohistochemistry results indicated that the rate of caspase-3 positive cells in control group was higher than that of the sham-operated group $(\mathrm{P}<0.01)$, while the rate of caspase- 3 positive cells in subgroups of the treatment group decreased compared to the control group, and the difference was statistically significant $(\mathrm{P}<0.01)$ (Figures $2 \mathrm{C}$ and 3 ).

\section{Apoptosis detection}

Apoptosis results showed that TUNEL-positive cells were significantly lower in the treatment group than the control group $(\mathrm{P}<0.01)$ (Figure 2D).

\section{Discussion}

A cerebral infarction is a type of ischemic stroke resulting from a blockage in the blood vessels supplying blood to the brain [7]. We 
established rat cerebral ischemia-reperfusion models to imitate cerebral infarction in this study. Cerebral ischemia-reperfusion injury is a complex pathophysiological process. Linnik confirmed that neuronal apoptosis involved in cerebral ischemia-reperfusion injury [8]. Therefore, preventing neuronal apoptosis can reduce the brain damage effectively. Recent studies have shown that the activation of caspase- 3 is a key step involved in neuron apoptosis after cerebral ischemia [9].

Currently, MSCs (mesenchyal stem cells) transplantation is prevalent in the field of cell therapy of cerebral infarction. Chen found that MSCs can significantly reduce neurological dysfunction in MCAO rats, but as he grew older, the amplification and differentiation of MSCs decreased significantly [10]. Recent studies have confirmed that MSCs also exist in UCB-MSCs, easy to access and no ethical issues. So we chose UCB-MSCs to treat cerebral ischemia-reperfusion injury in rats. This result indicated that the neurological function of UCB-MSCs treated rats improved significantly than the control group. However, the control group without UCB-MSCs injection improved slightly and it may be related to brain plasticity and natural restoration. And the number of neuronal apoptosis in the treatment group rat also decreased significantly compared to the control group. This shows that UCBMSCs play an important role in cerebral ischemia-reperfusion injury of the treatment rats.

Caspase-3, a homologous cysteine proteinase, also known as death protease, plays an important role in regulating cell apoptosis. Studies show that the expression level of caspase- 3 is low in normal adult rat brain tissues and increased significantly after cerebral ischemia [11,12]. Le also showed that the rate of TUNEL-positive cells decreased by $36 \%$ in cerebral ischemia-reperfusion rats compared with normal and caspase- 3 inhibitors can effectively reduce the number of apoptotic cells $[13,14]$. Therefore, growing evidence indicate that caspase-3 participates in cerebral ischemic injury and plays an important role in the neuronal apoptosis.

In conclusion, the present study found that UCB-MSCs improved the neurological function and reduced the transcription and translation level of caspase- 3 in cerebral ischemia-reperfusion rats, especially the number of apoptotic cells. These results provide a novel insight into the influence of the UCB-MSCs to cerebral infarction. However, the effects of UCB-MSCs on the caspase- 3 pathway and its therapeutic effect to cerebral infarction remain to be further explored.

\section{Acknowledgment}

This work was financially supported by National Nature Science Foundation (81471958).

\section{References}

1. Giroud M, Beuriat P, Becker F, Binnert D, Dumas R (1990) Dense middle cerebral artery: etiologic significance and prognosis. Rev Neurol (Paris) 146 : 224-227.

2. Patel DM, Shah J, Srivastava AS (2013) Therapeutic potential of mesenchymal stem cells in regenerative medicine. Stem Cells Int 2013: 496218.

3. Kolf CM, Cho E, Tuan RS (2007) Mesenchymal stromal cells. Biology of adult mesenchymal stem cells: regulation of niche, self-renewal and differentiation. Arthritis Res Ther 9: 204

4. Thalmeier K, Meissner P, Moosmann S, Sagebiel S, Wiest I, et al. (2001) Mesenchymal differentiation and organ distribution of established human stromal cell lines in NOD/SCID mice. Acta Haematol 105: 159-165.

5. Yu X, Chen D, Zhang Y, Wu X, Huang Z, et al. (2012) Overexpression of CXCR4 in mesenchymal stem cells promotes migration, neuroprotection and angiogenesis in a rat model of stroke. J Neurol Sci 316: 141-149.

6. Longa EZ, Weinstein PR, Carlson S, Cummins R (1989) Reversible middle cerebral artery occlusion without craniectomy in rats. Stroke 20: 84-91.

7. Ropper $\mathrm{AH}$ (2005) Adams and Victor's principles of neurology. McGraw-Hill Medical Pub Division, New York.

8. White BC, Sullivan JM, DeGracia DJ, O'Neil BJ, Neumar RW, et al. (2000) Brain ischemia and reperfusion: molecular mechanisms of neuronal injury. $J$ Neurol Sci 179: 1-33.

9. Graham SH, Chen J (2001) Programmed cell death in cerebral ischemia. J Cereb Blood Flow Metab 21: 99-109.

10. Chen J, Venkat P, Zacharek A, Chopp M (2014) Neurorestorative therapy for stroke. Front Hum Neurosci 8: 382.

11. Shimohama S, Tanino H, Fujimoto S (2001) Differential subcellular localization of caspase family proteins in the adult rat brain. Neurosci Lett 315: 125-128.

12. Harrison DC, Davis RP, Bond BC, Campbell CA, James MF, et al. (2001) Caspase mRNA expression in a rat model of focal cerebral ischemia. Brain Res Mol Brain Res 89: 133-146.

13. Karatas H, Aktas Y, Gursoy-Ozdemir Y, Bodur E, Yemisci M, et al. (2009) A nanomedicine transports a peptide caspase- 3 inhibitor across the blood-brain barrier and provides neuroprotection. J Neurosci 29: 13761-13769.

14. Le DA, Wu Y, Huang Z, Matsushita K, Plesnila N, et al. (2002) Caspase activation and neuroprotection in caspase-3-deficient mice after in vivo cerebral ischemia and in vitro oxygen glucose deprivation. Proceedings of the National Academy of Sciences 99: 15188-15193. 